\title{
Extracting hysteresis from nonlinear measurement of wavefront-sensorless adaptive optics system
}

\author{
H. Song, ${ }^{1, *}$ G. Vdovin, ${ }^{2}$ R. Fraanje, ${ }^{1}$ G. Schitter, ${ }^{1}$ and M. Verhaegen ${ }^{1}$ \\ ${ }^{1}$ Delft Center for Systems and Control, Delft University of Technology, Mekelweg 2, Delft, 2628 CD, The Netherlands \\ ${ }^{2}$ Flexible Optical B.V. Rontgenweg 1, Delft, 2624 BD, The Netherlands \\ *Corresponding author: h.song@tudelft.nl
}

Received September 12, 2008; revised November 7, 2008; accepted November 10, 2008;

posted November 24, 2008 (Doc. ID 101523); published December 24, 2008

\begin{abstract}
In many scientific and medical applications wavefront-sensorless adaptive optics (AO) systems are used to correct the wavefront aberration by optimizing a certain target parameter, which is nonlinear with respect to the control signal to the deformable mirror (DM). Hysteresis is the most common nonlinearity of DMs, which can be corrected if the information about the hysteresis behavior is present. We report a general approach to extract hysteresis from the nonlinear behavior of the adaptive optical system, with the illustration of a Foucault knife test, where the voltage-intensity relationship consists of both hysteresis and some memoryless nonlinearity. The hysteresis extracted here can be used for modeling and linearization of the AO system. (C) 2008 Optical Society of America
\end{abstract}

OCIS codes: $010.1080,230.6120$.

Deformable mirrors (DMs) with piezoelectric actuation are widely used in adaptive optics $(\mathrm{AO})$ systems to reduce the wavefront aberration [1,2]. However, the intrinsic hysteresis of the piezoactuators imposes a limit in the accuracy of the DM, which may degrade the convergence speed of the AO system or even introduce instability problems [1-4]. In conventional $\mathrm{AO}$ systems, the hysteresis of the piezo DM can be characterized with Shack-Hartman wavefront sensors, shearing interferometry or curvature sensors [5], etc., where the sensor output is linearly related to the actuator displacement or the curvature. But in wavefront-sensorless AO systems, which have been developing rapidly owing to their simplicity and low cost in implementation [6-8], piezo DM corrects the wavefront aberration by optimizing only a certain target parameter, such as the light intensity within a certain aperture, which suffers from some memoryless nonlinearity originating from the optical system as well as the hysteresis of the DM. Therefore we want to investigate how to extract the hysteresis of the piezo DM based only on the nonlinear measurement.

The schematic of the experimental setup is depicted in Fig. 1 (left). The collimated laser beam (He-Ne laser, with a wavelength of $632 \mathrm{~nm}$ ) passes through the beam splitter (BS) first, then is reflected by the DM and directed to the lens. Similar to the Foucault test [9], a razor blade blocks part of the beam at the back focal plane of the lens, followed by a photodiode (TSL250R-LF, TAOS, Korea) measuring the light intensity of the residual beam. The DM prototype (OKOTech, Delft, The Netherlands) has a clear aperture of $25 \mathrm{~mm}$ and the mirror plate is supported symmetrically by 12 piezoelectric tubes [PT130.00, Physik Instrumente, Germany; refer to Fig. 1 (right) for the actuator distribution]. The high voltage amplifier (HVA) has an input range of $0-5 \mathrm{~V}$ and a voltage amplification of 60 for frequencies lower than $1 \mathrm{kHz}$. Signal generation and data acquisition is accomplished by a dSPACE system (DS1103PPC, dSPACE, Germany) with the digital-to- analog card output range of $\pm 10 \mathrm{~V}, 14$ bits and the analog-to-digital card input range of $\pm 10 \mathrm{~V}, 16$ bits.

During each experiment, only one actuator in the DM is driven by the control voltage $V_{i}$ via the HVA, while the electrodes of all other actuators are open. Since the frequency of $V_{i}$ (less than $10 \mathrm{~Hz}$ ) is much lower than the first resonant frequency of the DM (about $1 \mathrm{kHz}$ ), all the dynamics can be neglected. The control voltage $V_{i}$ can be defined as the input of this optomechatronic system and the output is the light intensity measurement $V_{o}$. The problem is how to estimate the hysteresis of the piezoactuator based only on the $\left(V_{i}, V_{o}\right)$ data.

By physical modeling [10], $V_{o}$ can be represented as

$$
\begin{aligned}
V_{o}= & A \iint_{\Sigma} \mid \iint_{-\infty}^{+\infty} \exp \left[-j \frac{2 \pi}{\lambda}\left(\phi_{i}(\xi, \eta)+2 \phi_{m}(\xi, \eta)\right)\right] \\
& \times\left.\exp \left[-j \frac{2 \pi}{\lambda f}(u \xi+v \eta)\right] \mathrm{d} \xi \mathrm{d} \eta\right|^{2} \mathrm{~d} u \mathrm{~d} v
\end{aligned}
$$

where $A$ is a constant, $j=\sqrt{-1}, \lambda$ is the wavelength, and $f$ is the focal distance of the lens. $(\xi, \eta)$ and $(u, v)$ represent the coordinates in the input plane and the focal plane of the lens, respectively. $\Sigma$ represents the area in the focal plane that is not blocked by the razor blade. $\phi_{i}$ is the wavefront of the incident light. $\phi_{m}$ is the surface deflection of the DM, determined by the following equations [11]:

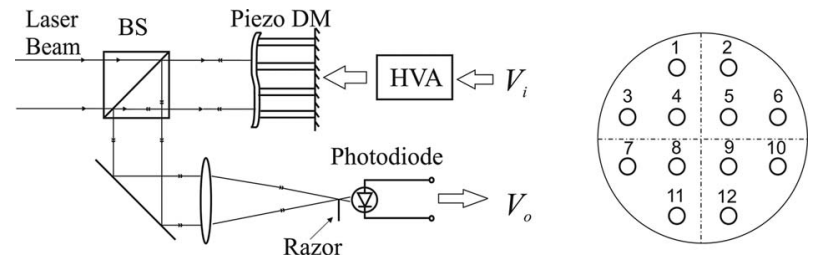

Fig. 1. Left, schematic of the experimental setup (input, control voltage $V_{i}$; output, light intensity measurement $V_{o}$ ). Right, distribution of the actuators in the DM. 


$$
\begin{gathered}
\phi_{m}(z)=\sum_{k=1}^{K} \frac{P_{k} W\left(z, \zeta_{k}\right)}{16 \pi D}+w_{0}+w_{1} z_{x}+w_{2} z_{y} \\
P_{k}= \begin{cases}k_{e}\left(h\left(V_{i}\right)-\phi_{m}\left(\zeta_{k}\right)\right) & \text { if actuator } k \text { is excited } \\
-k_{e} \phi_{m}\left(\zeta_{k}\right) & \text { otherwise }\end{cases} \\
\sum_{k=1}^{K} P_{k}=0, \quad \sum_{k=1}^{K} P_{k} \zeta_{k, x}=0, \quad \sum_{k=1}^{K} P_{k} \zeta_{k, y}=0
\end{gathered}
$$

where $z=\left(z_{x}, z_{y}\right)$ and $\zeta=\left(\zeta_{x}, \zeta_{y}\right)$ are the coordinates in the DM surface. $P_{k}, k=1 \ldots K$ are the forces applied to the DM faceplate by actuator $k$ at $\zeta_{k}$. $D, W\left(z, \zeta_{k}\right), w_{0}$, $w_{1}, w_{2}$, and $k_{e}$ are the coefficients. $h\left(V_{i}\right)$ represents the displacement of the actuator when driven by voltage $V_{i}$, with free end.

According to Mayergoyz's theory [3], hysteresis can be defined as the memory effect in the input-output relationship. This allows one to write Eqs. (1)-(4) as

$$
V_{o}=f(x), \quad x=H_{s}\left(V_{i}\right)
$$

where $H_{s}()$ represents the memory effect owing to hysteresis and $f()$ represents all memoryless nonlinearity originating from the optical system. $x$ denotes the output of $H_{s}()$. Since there is no other transformation in $H_{s}($ ) except for the memory, the hysteresis represented by $H_{s}()$ is normalized in the sense that two hysteresis branches in a hysteresis loop join at $\left(V_{i c}, x_{c}\right)$ with $x_{c}=V_{i c}$ (refer to Fig. 2), and this loop can be generalized for different input ranges.

To identify $H_{s}\left(\right.$ ) from $V_{i}$ and $V_{o}$, the signal $x$ needs to be reconstructed from $V_{o}$ as

$$
x=f^{-1}\left(V_{o}\right) \text {. }
$$

Physical expression of $f^{-1}()$ is difficult to achieve if only based on Eqs. (1)-(4), because the parameters such as $\phi_{i}$ and $\Sigma$ are unavailable in most practical cases. However, a numerical approach can be taken as an alternative, which consists of three steps:

1. Suppose there was no hysteresis in the system, then $x=V_{i}$. The virtual output $V_{o l}$ would be

$$
V_{o l}=f\left(V_{i}\right) .
$$

As hysteresis is present, $V_{o l}$ can be approximated by taking the average of the two branches in the $\left(V_{i}, V_{o}\right)$ loop. For a certain input $V_{i}=V_{i 0}$ (see Fig. 2), we get

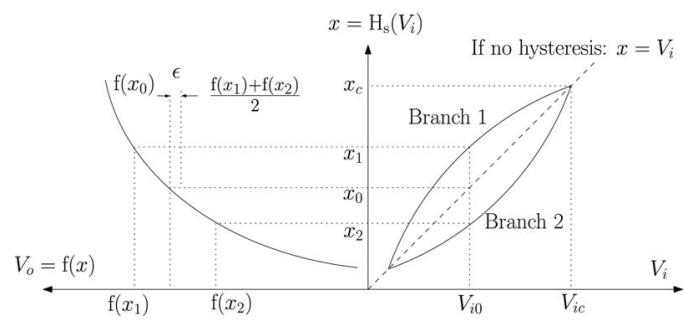

Fig. 2. Transfer from $V_{i}$ to $V_{o}$. Two hysteresis branches join at $x=V_{i}$. Virtual output $V_{o l}=f\left(x_{0}\right)=f\left(V_{i 0}\right)$ can be approximated by $\left[f\left(x_{1}\right)+f\left(x_{2}\right)\right] / 2$ with error $\epsilon$.

$$
\hat{V}_{o l}=\frac{1}{2}\left(f\left(x_{1}\right)+f\left(x_{2}\right)\right)=f\left(x_{0}\right)+\varepsilon,
$$

where $\hat{V}_{o l}$ is the approximation of $V_{o l}$ and

$$
\begin{aligned}
\varepsilon= & \left.\frac{1}{2} \frac{\mathrm{d} f(x)}{\mathrm{d} x}\right|_{x=x_{0}}\left(x_{1}+x_{2}-2 \cdot x_{0}\right) \\
& +\left.\frac{1}{2} \frac{\mathrm{d}^{2} f(x)}{2 ! \mathrm{d} x^{2}}\right|_{x=x_{0}}\left(\left(x_{1}-x_{0}\right)^{2}+\left(x_{2}-x_{0}\right)^{2}\right)+\ldots,
\end{aligned}
$$

is the approximation error. If $x_{1}-x_{0} \approx x_{0}-x_{2}$ and the higher-order terms are small enough, then $\hat{V}_{o l}$ $\approx f\left(x_{0}\right)=f\left(V_{i 0}\right)$. More generally,

$$
\hat{V}_{o l} \approx f\left(V_{i}\right) .
$$

2. The inverse of $f($ ) (if it exists), can be identified by polynomial curve fitting, defined as

$$
\min _{a_{m}}\left\|V_{i}-\hat{V}_{i}\right\|_{2}^{2}, \quad \text { with } \hat{V}_{i}=\hat{f}^{-1}\left(\hat{V}_{o l}\right)=\sum_{m=0}^{M} a_{m} \hat{V}_{o l}^{m},
$$

where $\hat{V}_{i}$ and $\hat{f}^{-1}()$ are the estimations of $V_{i}$ and $f^{-1}\left(\right.$ ), respectively. $M$ and $a_{m}$ are the order and the coefficients of the polynomial, respectively. If $f^{-1}()$ does not exist because the mapping from $\hat{V}_{o l}$ to $V_{i}$ is not unique, then the $\left(V_{i}, \hat{V}_{o l}\right)$ data set is divided into different subsets according to the first-order derivative of $\hat{V}_{o l}$ with respect to $V_{i}$ [12]. In each subset, the mapping from $\hat{V}_{o l}$ to $V_{i}$ is unique and thus can be approximated by function $\hat{f}_{n}^{-1}(), n=1,2 \ldots N$, where $N$ is the number of subsets.

3. Appropriate function $\hat{f}_{n}^{-1}()$ is selected for given $V_{o}$, according to the first-order derivative of $V_{o}$. Then the estimation of $x$, denoted by $\hat{x}$, is obtained by

$$
\hat{x}=\hat{f}_{n}^{-1}\left(V_{o}\right), \quad n=1,2 \cdots N .
$$

To improve the accuracy of $\hat{x}$, two methods can be employed: (1) $f\left(\right.$ ) and its inverse $f^{-1}()$ depend on the area $\Sigma$ [refer to Eq. (1)], and therefore the razor may be tuned such that $f^{-1}()$ is simple enough to be approximated accurately; (2) polynomials with higher order may be used to approximate $f^{-1}($ ).

Concerning the symmetry in the actuator layout and the optical system, during the experiments only actuator 1 or 4 is excited individually. Figure 3 shows the typical $\left(V_{i}, V_{o}\right)$ curves corresponding to actuator 1 (left) and 4 (right) when the razor is at different positions. The change of nonlinearity as well as the hysteresis can be observed clearly. To get accurate $\hat{x}$, only the curves with simple nonlinearity, marked as $A_{1}$, $A_{2}, B_{1}$, and $B_{2}$, are selected for further processing and the hysteresis curves extracted from these four are plotted in Fig. 4. To evaluate the accuracy of the estimation, hysteresis of a piezoactuator of the same type has been measured independently by a position sensor (S5990-01, HAMAMATSU, Japan) using an 

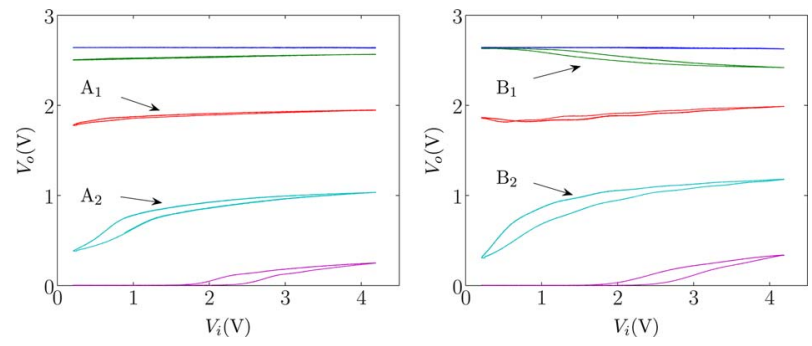

Fig. 3. (Color online) Left, $\left(V_{i}, V_{o}\right)$ curves for actuator 1 when the razor is at its initial position, tuned by $0.45,0.52$, 0.60 , and $0.80 \mathrm{~mm}$ (top to bottom). Right, $\left(V_{i}, V_{o}\right)$ curves for actuator 4 when the razor is at its initial position, tuned by $0.37,0.50,0.59$, and $0.80 \mathrm{~mm}$ (top to bottom).

optical lever, as shown in Fig. 4. Because the measured hysteresis curve involves linear transformations owing to the measurement as well as the memory owing to the hysteresis but $H_{s}()$ only includes the memory, the measured hysteresis curve has been scaled to eliminate the effect of linear transformations while keeping the memory unchanged. Table 1 shows the variance accounted for (VAF) for all $\hat{x}$ extracted from $A_{1}, A_{2}, B_{1}$, and $B_{2}$, with polynomial order $M=8,12,15$, where VAF is defined by

$$
\mathrm{VAF}=\left(1-\frac{\operatorname{var}\left(\hat{x}-x_{\text {measure }}\right)}{\operatorname{var}\left(x_{\text {measure }}\right)}\right) \times 100 \% \text {. }
$$

$x_{\text {measure }}$ is the hysteresis curve from the measurement after scaling and $\operatorname{var}(x)$ is the variance of $x$. Even in the worst case, VAF still reaches as high as $99.95 \%$, indicating quite accurate estimation of $x$.

Based on the hysteresis curve extracted from $A_{1}$ (with $M=8$, as in Fig. 4), an inverse ColemanHodgdon hysteresis model [1] has been identified and inserted between $V_{i}$ and the HVA to compensate for the hysteresis in the piezo DM. Figure 5 shows the resulting $\left(V_{i}, V_{o}\right)$ curves, where the reduction of hysteresis can be observed clearly compared with Fig. 3.
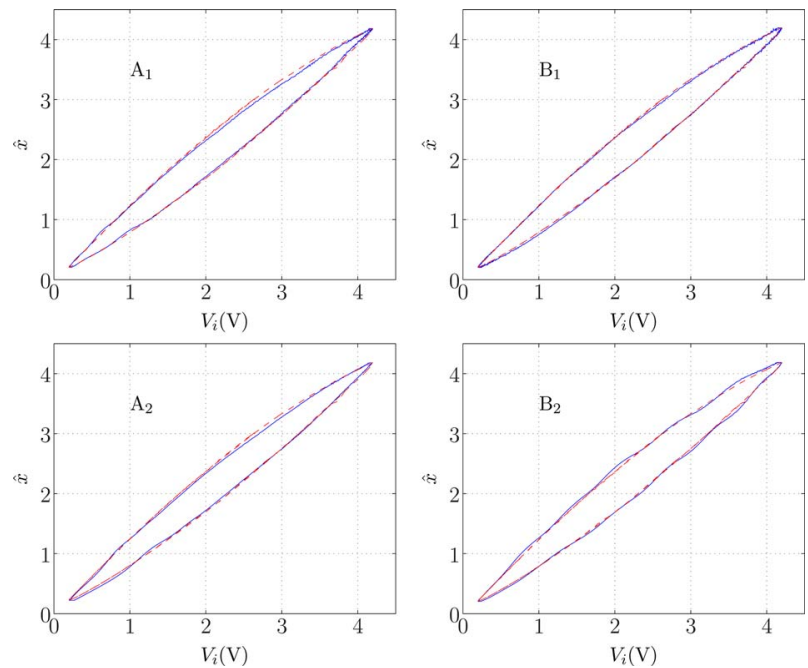

Fig. 4. (Color online) $\left(V_{i}, \hat{x}\right)$ curves (solid curve) extracted from $A_{1}, A_{2}, B_{1}$, and $B_{2}$, with polynomial order $M=8$. The hysteresis curve measured by a position sensor (dashed curve) is used for comparison.
Table 1. VAF of $\hat{x}$ Extracted from $A_{1}, A_{2}, B_{1}$, and $B_{2}$, with Polynomial Order $M=8,12,15$

\begin{tabular}{ccccc}
\hline$M$ & $A_{1}$ & $A_{2}$ & $B_{1}$ & $B_{2}$ \\
\hline 8 & $99.95 \%$ & $99.96 \%$ & $99.99 \%$ & $99.96 \%$ \\
12 & $99.95 \%$ & $99.96 \%$ & $99.99 \%$ & $99.97 \%$ \\
15 & $99.95 \%$ & $99.96 \%$ & $99.99 \%$ & $99.97 \%$ \\
\hline
\end{tabular}
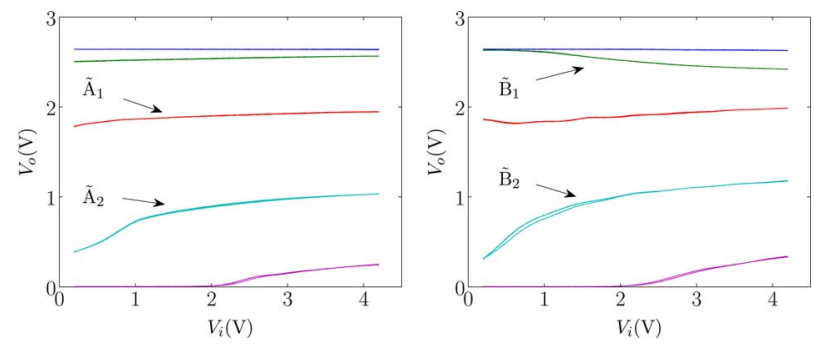

Fig. 5. (Color online) $\left(V_{i}, V_{o}\right)$ curves corresponding to actuator 1 (left) and 4 (right) after hysteresis compensation. The razor is at the same positions as in Fig. 3.

The largest gaps between two branches in $A_{1}, A_{2}, B_{1}$, and $B_{2}$ have been reduced by $67 \%(0.03$ to $0.01 \mathrm{~V})$, $89 \%(0.18$ to $0.02 \mathrm{~V}), 83 \%(0.06$ to $0.01 \mathrm{~V})$, and $86 \%$ $(0.21$ to $0.03 \mathrm{~V})$ as in $\widetilde{A}_{1}, \widetilde{A}_{2}, \widetilde{B}_{1}$, and $\widetilde{B}_{2}$, respectively, indicating accurate estimation of the hysteresis. Moreover, although the inverse hysteresis model is only based on the hysteresis curve extracted from $A_{1}$, it is still able to correct hysteresis in other conditions such as $A_{2}, B_{1}$, and $B_{2}$, which means good generalization property of the hysteresis estimation.

In conclusion, an experimentally proven general approach has been proposed to extract the hysteresis of a DM from the nonlinear measurement of the system target parameter, illustrated by our successful experiment with the Foucault knife test. The hysteresis extracted here can be used for modeling and linearization of the $\mathrm{AO}$ systems.

\section{References}

1. Q. Yang, C. Ftaclas, M. Chun, and D. Toomey, J. Opt. Soc. Am. A 22, 142 (2005).

2. A. Dubra, J. Massa, and C. Paterson, Opt. Express 13, 9062 (2005).

3. I. D. Mayergoyz, Mathematical Models of Hysteresis and Their Applications (Elsevier, 2003).

4. K. J. G. Hinnen, R. Fraanje, and M. Verhaegen, Proc. Inst. Mech. Eng. Part I - J. Systems Control Eng. 218, 503 (2004).

5. R. K. Tyson, Adaptive Optics Engineering Handbook (Dekker, 2000).

6. G. Vdovin, Proc. SPIE 3353, 902 (1998).

7. M. A. A. Neil, M. J. Booth, and T. Wilson, Opt. Lett. 25, 1083 (2000).

8. P. Marsh, D. Burns, and J. Girkin, Opt. Express 11, 1123 (2003).

9. L. Foucault, Annales de l'Observatoire imperial de Paris 5, 197 (1859).

10. J. W. Goodman, Introduction to Fourier Optics, 2nd ed. (McGraw-Hill, 1996).

11. M. Loktev, D. W. D. L. Monteiroa, and G. Vdovin, Opt. Commun. 192, 91 (2001).

12. G. Arfken, Mathematical Methods for Physicists, 3rd ed. (Academic, 1985). 